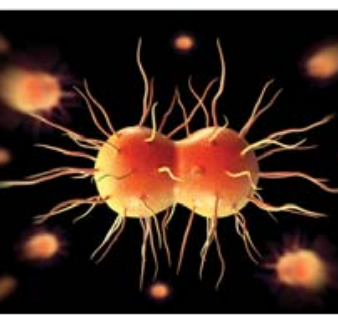

\title{
Screening tests for gonorrhoea should first do no harm
}

\author{
False-positive diagnosis causes important harms and should be minimised
}

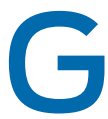

onorrhoea infection often has no clinical symptoms in women. Untreated, it may lead to pelvic inflammatory disease and carry risk of chronic pelvic pain, recurrent pelvic inflammatory disease, ectopic pregnancy and infertility. The risk of hospitalisation with pelvic inflammatory disease after gonorrhoea infection may be even greater than for chlamydia.

Preventing these complications is the rationale behind opportunistic screening. General practitioners are recommended to screen all sexually active Australians aged 15-29 years for chlamydia, but to screen only those at highest risk for gonorrhoea. ${ }^{2}$ However, as reported by Chow and colleagues in this issue of the Journal, ${ }^{3}$ gonorrhoea screening appears to have become increasingly common among all Australian women, including those at low risk. Two factors which may partly account for the increase in testing are (i) clinicians misinterpreting guidelines as meaning that opportunistic screening should be done for both infections, ${ }^{4}$ and (ii) use of dual nucleic acid amplification tests (NAATs) by laboratories to test for both infections, even when clinicians have only requested chlamydia testing.

As shown by Chow et al, the apparent recent increase in gonorrhoea incidence among Australian women is likely to be at least partly an artefact resulting from changes in testing practice. ${ }^{3}$ From 2008 to 2013, the number of Australian women being tested for chlamydia and gonorrhoea by NAAT increased, as did the number of gonorrhoea notifications among those tested by NAAT (which has a higher positive rate than culture). The authors contend that the true prevalence rate remained stable over the study period, and this is supported by stable rates within Melbourne Sexual Health Centre data (where diagnosis was by culture only) - especially if the population base for the clinic remained unchanged over time. These data argue against an increase in gonorrhoea prevalence but, because neither NAAT nor culture has optimum accuracy, there is uncertainty as to how many women have the disease.

In any case, even if all notifications (including those based on NAAT alone) are assumed to represent true rates of infection, the prevalence of gonorrhoea among non-Indigenous young Australians appears to be much less than that of chlamydia. Among young Indigenous Australians, however, there is a much higher prevalence of gonorrhoea compared with young non-Indigenous Australians, and this appears to be about equal to that of chlamydia. ${ }^{5}$ Screening for a condition which has a very low underlying prevalence, using a test with less than $100 \%$ specificity, is likely to lead to a large number of false-positive results.
The psychological consequences of false-positive test results are substantial. They include anxiety and depression, feelings of guilt and self-blame, loss of self-esteem and self-confidence, feelings of social isolation, and existential concerns.$^{6-8}$ In the case of mammography screening - perhaps the condition in which the impact of falsepositive diagnoses has been studied most — the adverse impact has been shown to last up to 3 years. ${ }^{9}$ Diagnosis of a sexually transmitted infection can also affect long-term sexual relationships, leading to concerns about trust and fidelity, and fear about disclosing results to a partner. ${ }^{8,10}$ There are physical consequences and adverse effects of treatment, ${ }^{9}$ and financial costs to the patient and the health care system. As is the case with many tests, the index case is not the only one affected - partners are notified, tested and potentially given inappropriate treatment. Hence it is widely recognised that all screening tests and programs should aim to minimise false-positive diagnoses. ${ }^{11}$

\section{"further tests (supplementary NAAT and culture) and repeat tests (eg, in 7 week) may be the best strategy for dealing with an initial positive NAAT result"}

The most obvious way to minimise false-positive diagnosis of gonorrhoea in Australia is to do as Chow et al (and guidelines) suggest: limit routine screening to higher-risk populations with higher underlying rates of infection. However, many laboratories may be unable to test for chlamydia and gonorrhoea separately, and will continue to test for both irrespective of what GPs request. Chow et al suggest that a possible solution to this is to suppress laboratories from reporting gonorrhoea results when they have not been requested by the clinician. ${ }^{3}$ This may be considered unacceptable practice by laboratory staff, however, who may prefer to use supplementary testing (or reflex testing) to confirm an initial positive result for gonorrhoea based on a NAAT. ${ }^{12}$ In such cases, supplementary testing involves an automatic algorithm being applied to initial test results such that a positive result for gonorrhoea based on a NAAT triggers testing with a second NAAT using a different target. Requiring both the initial NAAT and the supplementary NAAT to yield positive results before reporting a positive result would decrease the false-positive rate. This may not be enough in a very low-risk population, and repeat testing may be needed for assurance that the positive result is correct.

There remains the question of whether a NAAT (with supplementary testing) can be used as a stand-alone replacement for culture for diagnosing gonorrhoea infection, or 
whether it should only be used as part of a diagnostic pathway that includes culture - as a triage test (where culture is only done for NAAT-positive patients) and/ or an add-on test (where NAAT is done for high-risk patients who test negative on culture) ${ }^{13}$ Certainly, restricting NAATs to use as a triage test would decrease the false-positive rate compared with use of NAAT as a standalone replacement test without confirmatory culture. An additional argument against using NAAT as a replacement test is that we need to retain culture in the diagnostic pathway so that we can continue to monitor for possible antibiotic resistance. ${ }^{14}$ But, given the imperfect sensitivity of culture, using NAATs as an add-on test should be considered. ${ }^{15}$ When laboratory results differ or do not match the clinical picture, repeat testing is an option for deciding whether the patient has gonorrhoea infection.

To sort out these and related questions, we need data from well designed, prospective studies of high-risk populations. One such design is for all participants to have the initial NAAT and culture, with one or more further
NAATs (with different targets) done when the results of the first two tests differ. Until these types of studies have been done, we cannot determine the best screening and diagnostic testing pathway for gonorrhoea or estimate the true underlying rates of gonorrhoea infection in Australian populations. In the meantime, the takehome messages to primary care physicians are that (i) false-positive results are likely if a NAAT is used on its own in a low-risk population and (ii) further tests (supplementary NAAT and culture) and repeat tests (eg, in 1 week) may be the best strategy for dealing with an initial positive NAAT result.

Acknowledgements: This work was supported by funding from the National Health and Medical Research Council (Early Career Fellowship No. 1013390, Career Development Fellowship No. 1029241 and Program Grant No. 633003). We thank Bette Liu for her comments on a draft of this article.

Competing interests: No relevant disclosures.

Provenance: Commissioned; externally peer reviewed.

References are available online at www.mja.com.au. 
1 Reekie J, Donovan B, Guy R, et al. Hospitalisations for pelvic inflammatory disease temporally related to a diagnosis of chlamydia or gonorrhoea: a retrospective cohort study. PLOS ONE 2014; 9: e94361.

2 Royal Australian College of General Practitioners. Guidelines for preventive activities in general practice. 8th ed. Melbourne: RACGP, 2012. http://www.racgp.org.au/download/ Documents/Guidelines/Redbook8/redbook8.pdf (accessed Mar 2015).

3 Chow EPF, Fehler G, Read TRH, et al. Gonorrhoea notifications and nucleic acid amplification testing in a very low-prevalence Australian female population. Med J Aust 2015; 202: 321-323.

4 Hoad VC, Thambiran A. Evaluating the chlamydia and gonorrhoea screening program in the Humanitarian Entrant Health Service, Western Australia. Med J Aust 2012; 197: 47-49.

5 Graham S, Guy RJ, Donovan B, et al. Epidemiology of chlamydia and gonorrhoea among Indigenous and nonIndigenous Australians, 2000-2009. Med J Aust 2012; 197: 642-646.

6 McCaffery K, Waller J, Forrest S, et al. Testing positive for human papillomavirus in routine cervical screening: examination of psychosocial impact. BJOG 2004; 111: 1437-1443.

7 Dixon-Woods M, Stokes T, Young B, et al. Choosing and using services for sexual health: a qualitative study of women's views. Sex Transm Infect 2001; 77: 335-339.

8 Fortenberry JD, McFarlane M, Bleakley A, et al. Relationships of stigma and shame to gonorrhea and HIV screening. Am J Public Health 2002; 92: 378-381.
9 Brodersen J, Siersma VD. Long-term psychosocial consequences of false-positive screening mammography. Ann Fam Med 2013; 11: 106-115.

10 McCaffery K, Waller J, Nazroo J, Wardle J. Social and psychological impact of HPV testing in cervical screening: a qualitative study. Sex Transm Infect 2006; 82: 169-174.

11 Barratt A, Irwig L, Glasziou P, et al. Users guides to the medical literature: XVII. How to use guidelines and recommendations about screening. JAMA 1999; 281: 2029-2034.

12 Srivastava R, Bartlett WA, Kennedy IM, et al. Reflex and reflective testing: efficiency and effectiveness of adding on laboratory tests. Ann Clin Biochem 2010; 47: 223-227.

13 Bossuyt PM, Irwig L, Craig J, Glasziou P. Comparative accuracy: assessing new tests against existing diagnostic pathways. BMJ 2006; 332: 1089-1092.

14 Smith DW, Tapsall JW, Lum G. Guidelines for the use and interpretation of nucleic acid detection tests for Neisseria gonorrhoeae in Australia: a position paper on behalf of the Public Health Laboratory Network. Canberra: Australian Government Department of Health and Ageing, 2005. http:// www.health.gov.au/internet/main/publishing.nsf/Content/ cda-phln-gono-guidelines.htm (accessed Mar 2015).

15 Bromhead C, Miller A, Jones M, Whiley D. Comparison of the cobas 4800 CT/NG test with culture for detecting Neisseria gonorrhoeae in genital and nongenital specimens in a lowprevalence population in New Zealand. J Clin Microbiol 2013; 51: 1505-1509. 